\title{
Predicting complications of major head and neck oncological surgery: an evaluation of the ACS NSQIP surgical risk calculator
}

Peter S. Vosler, Mario Orsini, Danny J. Enepekides and Kevin M. Higgins

\begin{abstract}
Background: The American College of Surgeons National Surgical Quality Improvement Program (ACS NSQIP) universal surgical risk calculator is an online tool intended to improve the informed consent process and surgical decision-making. The risk calculator uses a database of information from 585 hospitals to predict a patient's risk of developing specific postoperative outcomes.
\end{abstract}

Methods: Patient records at a major Canadian tertiary care referral center between July 2015 and March 2017 were reviewed for surgical cases including one of six major head and neck oncologic surgeries: total thyroidectomy, total laryngectomy, hemiglossectomy, partial glossectomy, laryngopharyngectomy, and composite resection. Preoperative information for 107 patients was entered into the risk calculator and compared to observed postoperative outcomes. Statistical analysis of the risk calculator was completed for the entire study population, for stratification by procedure, and by utilization of microvascular reconstruction. Accuracy was assessed using the ratio of predicted to observed outcomes, Receiver Operating Characteristics (ROC), Brier score, and the Wilcoxon signed-ranked test.

Results: The risk calculator accurately predicted the incidences for 11 of 12 outcomes for patients that did not undergo free flap reconstruction (NFF group), but was less accurate for patients that underwent free flap reconstruction (FF group). Length of stay (LOS) analysis showed similar results, with predicted and observed LOS statistically different in the overall population and FF group analyses ( $p=0.001$ for both), but not for the NFF group analysis $(p=0.764)$. All outcomes in the NFF group, when analyzed for calibration, met the threshold value (Brier scores $<0.09$ ). Risk predictions for 8 of 12 , and 10 of 12 outcomes were adequately calibrated in the FF group and the overall study population, respectively. Analyses by procedure were excellent, with the risk calculator showing adequate calibration for 7 of 8 procedural categories and adequate discrimination for all calculable categories (6 of 6).

Conclusion: The NSQIP-RC demonstrated efficacy for predicting postoperative complications in head and neck oncology surgeries that do not require microvascular reconstruction. The predictive value of the metric can be improved by inclusion of several factors important for risk stratification in head and neck oncology.

Keywords: National surgical quality improvement program risk calculator, Risk assessment, Head and neck, Cancer, Brier score, Surgical complications, Outcome measures, Free flap reconstruction, Microvascular reconstruction

\footnotetext{
* Correspondence: Kevin.Higgins@sunnybrook.ca

Department of Otolaryngology—Head \& Neck Surgery, Sunnybrook Health

Sciences Centre, University of Toronto, 2075 Bayview Avenue, Suite M1 102,

Toronto, ON M4N 3M5, Canada
} 


\section{Background}

Identifying patients at increased risk for perioperative complications imperative for determining candidates for surgical intervention and proper preoperative patient counselling. This is particularly relevant in the head and neck oncology discipline where complications such as fistula development and free flap necrosis can result in significantly extended length of stay and decreased quality of life. Surgery-specific risk calculators are available for various surgical practices [1-4], and risk factors for cardiovascular and head and neck-specific complications for head and neck procedures including duration of anaesthesia and ASA are known $[5,6]$; however, there are no head and neck-specific risk calculators available.

The universal surgical risk calculator created by the American College of Surgeons National Surgical Quality Improvement Program (ACS NSQIP) was developed as both a surgical aid and informed consent tool to improve the overall decision-making process. The risk calculator is an open-access online tool that uses an algorithm and validated data from over 500 hospitals and 2.7 million operations performed in the Unites States to predict the likelihood of 12 postoperative outcomes [7-11]. This online program accepts the input of 20 comorbidity and demographic-related, patient-specific variables, (such as age group, gender, smoking status, functional status, body-mass index, etc.) in conjunction with a surgery-specific Current Procedure Terminology (CPT) code, to predict patients' risk of 12 postoperative outcomes within 30 days after surgery $[12,13]$. Patient-specific variables chosen for the risk calculator are broadly applicable for patients undergoing one of the 1887 procedures covered [11, 13, 14].

Recently, this system has been evaluated in several surgical subspecialties including head and neck surgery [8, 15-17], urology [9], neurology [10], pulmonology [18], gynecology [19], gastroenterology [12, 20-23], orthopedics [24] and general surgery with variable results. Despite these numerous publications, very few studies have examined the risk calculator's efficacy in a Canadian setting $[13,14]$ and none, so far, have evaluated head and neck surgery in a Canadian setting. We aim to provide the first comprehensive evaluation of the risk calculator for head and neck surgery in a Canadian setting by assessing its accuracy in predicting complications following six major head and neck procedures.

Our study evaluates the ACS NSQIP surgical risk calculator for predictive accuracy by comparing forecasted 30day postoperative outcomes to observed incidences for patients who underwent one of six head and neck surgeries: total thyroidectomy, total laryngectomy, hemiglossectomy, partial glossectomy, laryngopharyngectomy and composite resection. These representative procedures were chosen for review because they are frequently performed by head and neck surgeons (thyroidectomy) or are associated with increased complexity and resultant higher complication rates and average length of stay (composite resection, laryngopharyngectomy). Separate statistical analyses were completed for the overall study population, and for each individual procedure. Furthermore, stratification and statistical analysis by free flap utilization, where patients were either separated into the Free Flap reconstructed (FF) group or Non-Free Flap reconstructed (NFF) group, was completed. This sub-stratification was warranted due to the risk calculator lacking separate CPT codes for head and neck procedures that included free flap reconstruction. This is an important distinction because it is wellestablished that free flap reconstruction increases patients' time under anesthesia, and it increases risk of surgical site infection and donor site complications $[5,25,26]$.

\section{Methods \\ Study design}

A retrospective review of all patients that underwent head and neck surgery at a single Canadian tertiary care referral center, between July 2015 and March 2017 was completed following research ethics board approval. Procedures included for review were total thyroidectomy, total laryngectomy, hemiglossectomy, partial glossectomy, laryngopharyngectomy and composite resection. Each patient's demographic-, procedure-, and complication- related data were collected and maintained in a secure database.

Demographic information was manually entered into the ACS NSQIP surgical risk calculator [25] to fill 20 patient-specific risk factors including age group, gender, functional status, BMI, ASA class, hypertension, smoking status and COPD. The most relevant Current Procedural Terminology (CPT) codes were then selected based on the type, extent, and attributes of the procedure; the following guidelines were used, hemiglossectomy $=41135$, partial glossectomy $=41120$, laryngectomy $=31365$, laryngopharyngectomy with free flap $=31395$, composite resection with free flap $=41153$ or 41155 , thyroidectomy with central neck dissection $=60252$, thyroidectomy without neck dissection $=60240$. To maintain consistency, "Surgeon Adjustment of Risk" was not altered.

Patient-specific estimates of postoperative risk, determined by the risk calculator, including serious complications, any complications, pneumonia, cardiac complications, surgical site infection (SSI), urinary tract infection (UTI), venous thromboembolism (VTE), renal failure, readmission, discharge to nursing or rehabilitation facility, return to operating room (ROR), and death, were recorded. Complications were simply tallied, with no additional weight given to multiple complications in the same patient. Observed incidences of complication as determined by database review were compared with the risk calculator's predictions. 


\section{Statistical analysis}

Statistical analyses were completed for the overall study population, as well as for subset analysis by procedure type, and by presence or absence of free flap reconstruction (FF group and NFF group, respectively). Predicted and observed rates of incidences were then compared within each stratification. Brier score, ROC curves, likelihood ratios and Wilcoxon signed-ranked tests for nonparametric data were generated with SPSS, version 24.0.

\section{Discrimination - ROC curves}

Evaluation of the ACS NSQIP surgical risk calculator for discrimination ability was completed using area under (AUC) the receiver operating characteristics (ROC) curve, sometimes called c-statistic. Receiver operating characteristics curve graphs model sensitivity (true positive predictions) against 1 - specificity (false positive predictions) to evaluate how well a model distinguishes between higher and lower risk units within a population. The area under this graph is considered an accurate representation of a model's discrimination and is scored between 1.0 to 0.5 , where the former is considered perfectly predictive and the latter is equivalent to chance. C-statistic values $>0.7$ are considered adequately discriminative whereas values of $>0.8$ are considered strongly discriminative $[9,10,26,27]$.

\section{Calibration - brier score}

Calibration is a measure of how well a model's predictions 'fit' an observed incidence rate over a collection of predictions [10]. The Brier score, which is the sum of the mean squared differences between predicted values and binary outcomes, was used to evaluate the ACS NSQIP surgical risk calculator for calibration. Incidences were given a value of 1 and non-incidences were given a value of 0 . Brier score values range from 0.0 to 1.0 , where smaller values represent increased calibration and accuracy. Several thresholds, varying from 0.01 to 0.16 , have been suggested to quantify sufficient accuracy for the Brier score $[16,17,19,21] ; 0.09$ was used as it is considered accurate for outcomes with low incidences $[28,29]$.

\section{Results}

\section{Patient characteristics}

Patients that underwent operations at a single, tertiary, Canadian teaching hospital between July 2015 and March 2017 were retrospectively reviewed for the purposes of this study. A total of 131 patients underwent operations, and 24 patients lacking adequate records or without 30-day follow-up information were excluded from the study, leaving a final population size of 107 individuals. Twenty-seven postoperative complications were observed within 30 days of surgery for the patients in this study (25\%). The patient group had a mean age of 61 years, was composed approximately evenly of males and females (54, 53 years, respectively) and was, on average, overweight (Body Mass Index $=27.6 \mathrm{mg} /$ $\left.\mathrm{kg}^{2}\right)$. Most patients were non-diabetic (87\%) and exhibited a high incidence of hypertension (40\%). There were no emergency cases $(0 \%)$, and one patient was on dialysis before their operation (1\%). The most commonly observed postoperative complications were ROR (12 incidences, 11\%), SSI (11 incidences, 10\%) and readmission (5 incidences, 5\%). A full outline of demographic information can be found in Table 1 .

\section{Predicted vs. observed outcomes}

Summary analyses demonstrating total observed and predicted incidences of complications without stratification were calculated (Fig. 1). The risk calculator exhibited $100 \%$ accuracy in predicting renal failure and adequate performance in predicting readmission (predicted $=6$, observed $=5$ ) and ROR (predicted $=9$, observed $=12$ ). However, it underestimated serious complications (35\%), any complication (30\%), cardiac complications (100\%), SSI (36\%), UTI (50\%), and ROR (25\%). Overestimations were equally common with pneumonia (50\%), VTE, readmission $(20 \%)$, death, and discharge to nursing and rehabilitation (133\%), all scoring below predicted.

Stratification of the data based on use of free flap reconstruction revealed that the NSQIP- risk calculator was accurate in patients that did not receive microvascular reconstruction. Figure 2 depicts the stratification of predicted complications as calculated by the ACS NSQIP surgical risk calculator compared to observed incidences for those patients who underwent free flap reconstruction $(n=58)$ and those that did not $(n=49)$. The risk calculator correctly predicted the number of complications in the NFF patient group for 11 of 12 outcomes, but exhibited a $100 \%$ overestimation for the remaining outcome-readmission (predicted $=2$, observed $=1$ ). For the patient cohort that underwent free flap reconstruction, the risk calculator demonstrated more limited accuracy, predicting only renal failure (predicted $=0, \quad$ observed $=0), \quad$ readmission $\quad($ predicted $=4$, observed $=4$ ) and death (predicted $=0$, observed $=0$ ), within acceptable error. Six of the remaining nine outcomes were underestimated, while the rest were overestimated.

\section{Free flap vs. no free flap}

In our population, 58 (54\%) patients underwent free flap reconstruction as part of their procedure. $\mathrm{C}$-statistic and Brier score analyses were calculated for each of these stratifications. Overall, the risk calculator demonstrated improved calibration and comparable discrimination in predicting complications for the non-free flap reconstructed group when compared to the free flap reconstructed group (Table 2). Values shown in bold are 
Table 1 Demographic and Medical Information

\begin{tabular}{|c|c|}
\hline Characteristic & $\begin{array}{l}\text { Value } \\
(n=107)\end{array}$ \\
\hline Age, mean (SD), y & $61.1(13.4)$ \\
\hline Male gender, \# (\%) & $54(50)$ \\
\hline Height, mean (SD), m & $1.65(0.14)$ \\
\hline Weight, mean (SD), kg & $74.1(17.2)$ \\
\hline BMl, mean (SD) & $27.6(6.65)$ \\
\hline Underweight, \# (\%) & $5(5)$ \\
\hline Normal weight, \# (\%) & $29(27)$ \\
\hline Overweight, \# (\%) & $29(27)$ \\
\hline Obese, \# (\%) & $23(21)$ \\
\hline Unknown, \# (\%) & $21(20)$ \\
\hline \multicolumn{2}{|l|}{ Functional Status, \# (\%) } \\
\hline Independent & $106(99)$ \\
\hline Partially Dependent & $1(1)$ \\
\hline Dependent & $0(0)$ \\
\hline \multicolumn{2}{|l|}{ ASA Class, \# (\%) } \\
\hline । & $26(24)$ \\
\hline$\|$ & $54(50)$ \\
\hline III & $27(25)$ \\
\hline IV & $0(0)$ \\
\hline \multicolumn{2}{|l|}{ Diabetes, \# (\%) } \\
\hline No & $93(87)$ \\
\hline Oral Medication & $4(4)$ \\
\hline Insulin & $10(9)$ \\
\hline \multicolumn{2}{|c|}{ Hypertension requiring medication, \# (\%) } \\
\hline Yes & $43(40)$ \\
\hline No & $64(60)$ \\
\hline \multicolumn{2}{|l|}{ Dyspnea, \# (\%) } \\
\hline No & $92(86)$ \\
\hline With moderate exertion & $12(11)$ \\
\hline At rest & $3(3)$ \\
\hline \multicolumn{2}{|c|}{ Current Smoker (within 1 year), \# (\%) } \\
\hline Yes & $23(21)$ \\
\hline No & $84(79)$ \\
\hline \multicolumn{2}{|l|}{ History of severe COPD, \# (\%) } \\
\hline Yes & $13(12)$ \\
\hline No & $94(88)$ \\
\hline \multicolumn{2}{|l|}{ Dialysis, \# (\%) } \\
\hline Yes & $1(1)$ \\
\hline No & $106(99)$ \\
\hline \multicolumn{2}{|c|}{ Steroid use for chronic condition, \# (\%) } \\
\hline Yes & $8(7)$ \\
\hline No & $99(93)$ \\
\hline Emergency case: Yes, \# (\%) & $0(0)$ \\
\hline
\end{tabular}

Table 1 Demographic and Medical Information (Continued)

\begin{tabular}{ll}
\hline Characteristic & $\begin{array}{l}\text { Value } \\
(n=107)\end{array}$ \\
\hline Ascites within 30 days prior to surgery: Yes, \# (\%) & $0(0)$ \\
Sepsis within 48 h: Yes, \# (\%) & $0(0)$ \\
Acute renal failure: Yes, \# (\%) & $0(0)$ \\
Use of mechanical ventilation: Yes, \# (\%) & $0(0)$ \\
Congestive Heart Failure 30 days prior to surgery: Yes, \# (\%) & $0(0)$ \\
\hline
\end{tabular}

considered to meet or exceed the threshold values for their respective test statistics. Twelve of twelve Brier score values in the NFF group were below threshold $(0.09)$ and 3 of 12 c-statistic values reached adequacy (0.70). Conversely, in the FF group, only 8 of 12 Brier score values showed acceptable calibration and 4 of 12 c-statistic values scored above 0.70. Specifically, SSI (Brier $=0.019$, $\mathrm{ROC}=0.885)$, readmission $($ Brier $=0.018$, ROC $=1.00$ ), and discharge to nursing or rehabilitation (Brier $=0.023$, ROC $=0.854$ ) exhibited adequate discrimination and calibration in the NFF group, and pneumonia $($ Brier $=0.017, R O C=0.912$ ), cardiac complication $($ Brier $=0.051, \mathrm{ROC}=0.767)$, readmission $($ Brier $=0.063$, ROC $=0.713$ ), and discharge to nursing or rehabilitation (Brier $=0.028, \mathrm{ROC}=0.987$ ) scored adequately in the FF group. C-statistic values shown as 'N/A' were incalculable due to an absence of observed complications for those outcomes.

When considering the entire study population without stratification by free flap, the risk calculator benefited from the effects of increased heterogeneity on discrimination, with 6 of 12 outcomes scoring above 0.70 . Outcomes that scored satisfactorily for both discrimination and calibration were: cardiac complication $(\mathrm{Brier}=0.028$, ROC $=0.838)$, SSI (Brier $=0.088, \quad$ ROC $=0.790), \quad$ ROR (Brier $=0.087, \mathrm{ROC}=0.750$ ), and discharge to nursing or rehabilitation (Brier $=0.026, \mathrm{ROC}=0.851$ ). Two of the remaining outcomes, serious complications and any complications, scored acceptably for discrimination ( $\mathrm{ROC}=0.707$ and 0.719 , respectively), but inadequately for calibration (Brier $=0.174$ and 0.174 , respectively). Conversely, pneumonia, UTI, and readmission met calibration requirements $($ Brier $=0.019,0.018$, and 0.045, respectively), but did not score above the threshold for discrimination ( $\mathrm{ROC}=0.543,0.498$, and 0.604 , respectively). Incidences of death, venous thromboembolism or renal failure were not observed; thus, c-statistics for these outcomes could not be calculated.

\section{Stratification by procedure}

Evaluation of the risk calculator was also stratified by procedure (Table 3) where patients were separated into one of the following groups: hemiglossectomy with free flap, partial glossectomy, laryngectomy, laryngopharyngectomy 
with free flap, composite resection with free flap, and thyroidectomy. Thyroidectomy was further subdivided into procedures with central neck dissection and those without. Table 3 indicates the number of patients in each stratum, the percentage of patients with stage IV cancer for cases with available staging information $(n=89)$, and the instances of preoperative radiation. One patient was excluded from analysis as a result of procedural characteristics not adequately fitting into the above groups. The occurrences of preoperative radiation (Likelihood Ratio $=$ 21.689, $p=0.003$ ) and the incidences of stage IV cancer (Likelihood Ratio $=49.314, p=0.001$ ) were significantly different between groups. This is to be expected considering the different standards of care associated with different disease locations and extensions. The risk calculator demonstrated adequate calibration and discrimination for laryngopharyngectomy with free flap $(\mathrm{Brier}=0.073, \mathrm{ROC}=$ 0.842 ), composite resection with free flap (Brier $=0.081$, ROC $=0.842$ ), thyroidectomy overall $($ Brier $=0.024$, $\mathrm{ROC}$ $=0.817$ ), and thyroidectomy with central neck dissection (Brier $=0.023, \mathrm{ROC}=0.816)$ or without central neck dissection (Brier $=0.027$, ROC $=0.845$ ). The risk calculator's ability to predict complications secondary to laryngectomy showed excellent discrimination $(\mathrm{ROC}=0.956)$ but fell just short of acceptable calibration (Brier $=0.095$ ). The remaining procedures, hemiglossectomy with free flap and partial glossectomy, exhibited acceptable calibration (Brier $=0.003$ and 0.001, respectively) but, due to lack of observed complications, had incalculable c-statistics. Overall, the risk calculator performed exceptionally well in predicting complications by procedure; however, this is biased by the few patients in most of the groups.

\section{Overall}

The ACS NSQIP surgical risk calculator's overall discrimination and calibration scores were calculated by evaluating all predictions of complications (12 predictions for each patient) together; this was completed for both FF and NFF groups, as well as, for the entire study population (Table 4). In all three instances the risk calculator exhibited accurate discrimination and calibration: FF $(\mathrm{Brier}=0.081, \mathrm{ROC}=$ $0.85)$, NFF (Brier $=0.023, \quad \mathrm{ROC}=0.78$ ), and combined (Brier $=0.055, \mathrm{ROC}=0.86$ ).

\section{Length of stay}

Length of stay analyses were completed for the overall study population and for both NFF and FF stratifications. Analyses were completed using the Wilcoxon signed-ranked test for nonparametric data $[12,22]$ and one patient was excluded from analysis due to ongoing hospital stay at time of manuscript development. Figure 3 compares the box plots of observed and predicted lengths of stay for each sub-stratification and the total study population. Predicted lengths of stay for the total study population and for the FF stratified group were found to be significantly different than observed lengths of stay $(p=0.001)$. Conversely, predicted length of stay for the NFF stratified group was not found to be significantly different from observed length of stay $(p=0.764)$.

\section{Discussion}

Improving the informed consent process, providing surgeons with essential preoperative information, and acting as a quality control measure, are all potential benefits obtainable from an accurate surgical risk calculator. For these reasons, and others, various subspecialties have evaluated the efficacy of the ACS NSQIP surgical risk calculator in practice. Results from these studies are markedly inconsistent, with conclusions for the risk calculator ranging from entirely non-predictive for all outcomes [9] to useful within judicious parameters [22].

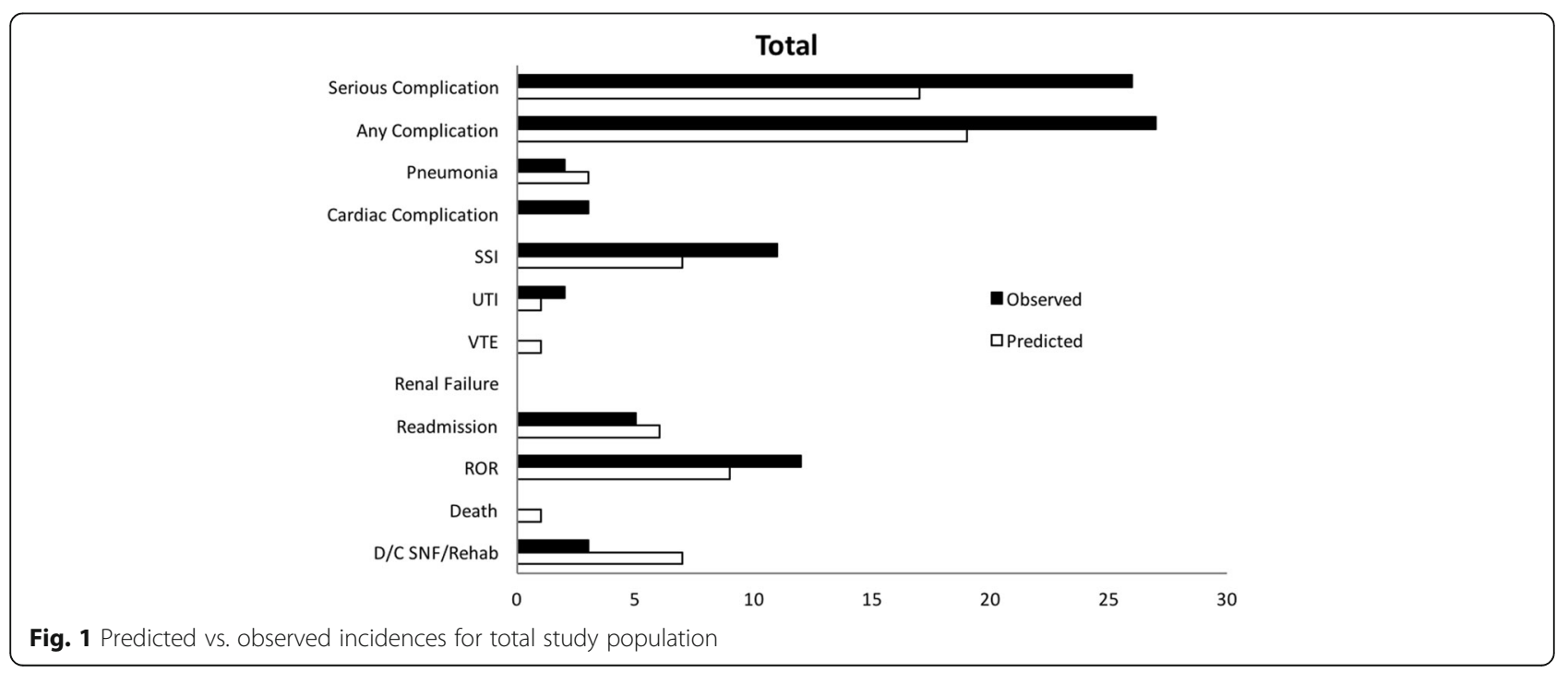




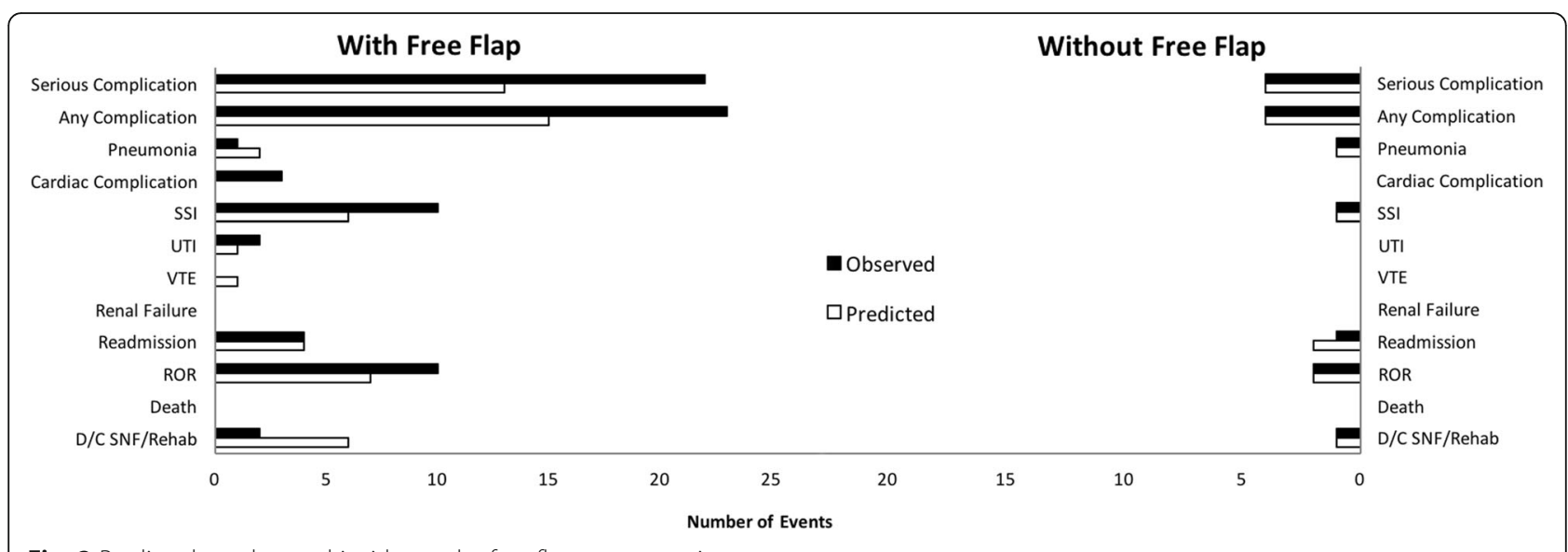

Fig. 2 Predicted vs. observed incidences by free flap reconstruction

One potential cause for these varying results is lack of agreement on a threshold value for the Brier Score. Several studies consider scores below 0.01 as predictive [9, 16], referencing Bilimoria et al. and Cohen et al. as justification for this decision. Bilimoria et al., however, does not use a Brier score threshold of 0.01 in their publication and interestingly, considered a value of 0.069 as sufficiently accurate [30]. Additionally, Cohen et al. does not at all use, nor refer to, the Brier score test in their analysis [31]. Other published threshold values are much higher, including 0.16 [21] and 0.14 [22]. Due to these discrepancies, we decided to use 0.09 , a relatively intermediate value that has been shown to indicate accuracy for models with low incidence rates $[28,29]$.

Some studies have found that the surgical risk calculator systemically underestimates the true risk of postoperative complications [20, 32]. Lack of surgery-specific metrics and predicted outcomes inherent to the risk calculator are often implicated as a cause of this inaccuracy [32]. For this reason, many studies have tested the validity of supplementary specialty-specific preoperative metrics to support the 21 already captured by the ACS NSQIP surgical risk calculator program [12, 23, 33]. As opposed to evaluating additional head and neck -specific preoperative metrics, we decided stratification by surgical complexity, free flap reconstruction versus no free flap reconstruction, and surgical type, as was previously unstudied, could delineate the risk calculator's accuracy as a function of surgical complexity. Initial evaluation of the risk calculator for observed versus predicted events, without stratification, exhibited results similar to other studies with 5 of 12 and 6 of 12 outcomes overestimated $[9,23]$ and underestimated, respectively. However, upon stratification by free flap utilization, the primary driver of these inaccuracies was

Table 2 Brier score and ROC AUC by Risk Calculator outcome

\begin{tabular}{|c|c|c|c|c|c|c|}
\hline \multirow[b]{2}{*}{ Outcome } & \multicolumn{2}{|c|}{ Without Free Flap ${ }^{*}$, ,a } & \multicolumn{2}{|c|}{ With Free Flap ${ }^{*},+, a$} & \multicolumn{2}{|c|}{ Combined ${ }^{*}, t, a$} \\
\hline & Brier Score & $\mathrm{ROC}$ & Brier Score & $\mathrm{ROC}$ & Brier Score & $\mathrm{ROC}$ \\
\hline Serious Complications & 0.0838 & 0.5220 & 0.2508 & 0.6060 & 0.1743 & 0.7070 \\
\hline Any Complications & 0.0861 & 0.5500 & 0.2474 & 0.6280 & 0.1735 & 0.7190 \\
\hline Pneumonia & 0.0208 & 0.3230 & 0.0171 & 0.9120 & 0.0188 & 0.5430 \\
\hline Cardiac Complications & 0.0000 & N/A & 0.0509 & 0.7670 & 0.0276 & 0.8380 \\
\hline SSI & 0.0193 & 0.8850 & 0.1453 & 0.6830 & 0.0876 & 0.7900 \\
\hline UTI & 0.0001 & N/A & 0.0339 & 0.4420 & 0.0184 & 0.4980 \\
\hline VTE & 0.0001 & N/A & 0.0002 & N/A & 0.0001 & N/A \\
\hline Renal Failure & 0.0000 & N/A & 0.0000 & N/A & 0.0000 & $\mathrm{~N} / \mathrm{A}$ \\
\hline Readmission & 0.0183 & 1.0000 & 0.0627 & 0.7130 & 0.0445 & 0.6040 \\
\hline ROR & 0.0234 & 0.2190 & 0.1406 & 0.6880 & 0.0869 & 0.7500 \\
\hline Death & 0.0000 & N/A & 0.0001 & $\mathrm{~N} / \mathrm{A}$ & 0.0001 & $\mathrm{~N} / \mathrm{A}$ \\
\hline D/C SNF/Rehab & 0.0232 & 0.8540 & 0.0281 & 0.9870 & 0.0259 & 0.8510 \\
\hline
\end{tabular}

*Brier scores of $<0.09$ and ROC $>0.7$ (bold values) were considered accurate

${ }^{a} \mathrm{~N} / \mathrm{A}$ indicates no ROC score could be calculated due to the absence of complications

${ }^{\dagger}$ Bold values meet or exceed their respective accuracy thresholds 
Table 3 Brier Score, ROC AUC, and demographic information by procedure type

\begin{tabular}{|c|c|c|c|c|c|c|}
\hline Procedure & $\mathrm{N}$ & Brier Score, ${ }^{*}$ & $\mathrm{ROC}^{\mathrm{a}, \mathrm{t}}$ & $\% \mathrm{~T} 4 \mathrm{a}$ & Radiated Patients & CPT Code(s) \\
\hline Hemiglossectomy with Free Flap & 4 & 0.0032 & N/A & $25 \%$ & 0 & 41,135 \\
\hline Partial Glossectomy & 12 & 0.0015 & N/A & $0 \%$ & 0 & 41,120 \\
\hline Laryngectomy & 5 & 0.0954 & 0.956 & $100 \%$ & 3 & 31,365 \\
\hline Laryngopharyngectomy with Free Flap & 16 & 0.0732 & 0.842 & $81 \%$ & 2 & 31,395 \\
\hline Composite Resection with Free Flap & 39 & 0.0807 & 0.842 & $30 \%$ & 4 & $41,153 \& 41,155$ \\
\hline Thyroidectomy Overall & 30 & 0.0241 & 0.817 & $7 \%$ & 0 & $60,252 \& 60,240$ \\
\hline Thyroidectomy with neck dissection & 21 & 0.0229 & 0.816 & $7 \%$ & 0 & 60,252 \\
\hline Thyroidectomy without neck dissection & 9 & 0.0273 & 0.845 & $0 \%$ & 0 & 60,240 \\
\hline
\end{tabular}

*Brier scores of $<0.09$ and ROC $>0.7$ (bold values) were considered accurate

${ }^{a} \mathrm{~N} / \mathrm{A}$ indicates no ROC score could be calculated due to the absence of complications

${ }^{+}$Bold values meet or exceed their respective accuracy thresholds

elucidated. In the FF group, the risk calculator over- or under-predicted 9 of 12 outcomes. In the NFF group, however, outcomes were exceptionally well predicted, with 11 of 12 complications accurately projected by the risk calculator. We believe increased technical difficulty, potential sacrifice of vital structures (i.e. internal jugular vein, spinal accessory nerve, phrenic nerve, sympathetic trunk, and the thoracic duct), longer duration of anaesthesia, and extended post-operative stay, are potential contributors to the disparity between these stratifications.

Statistical results similar to that of predicted and observed outcomes was demonstrated with LOS; length of stay predictions for the overall population and FF subgroup were found to significantly differ from observed postoperative stays. However, when LOS analysis was completed for the NFF stratified group, predicted and observed stays were not found to significantly differ. Furthermore, Fig. 3 clearly depicts the dissimilarity between predicted and observed LOS for the FF group and overall population, when compared to that of the NFF group. Our overall population and the FF sub-group results are consistent with many other publications, which found little to no association between observed and predicted length of stay [12, 15-17, 21, 22]. Massoumi et al. implicated the level of care offered by a hospital (primary vs. tertiary) and its practices as potential generators of these inconsistencies [20].

Our results follow a general trend present in the literature: increasingly complex procedures, where postoperative outcomes are closely influenced by the case-by-case features of the procedure, the skill level of the surgeon and postoperative care practices, are more likely miss- projected by the ACS NSQIP risk calculator, which cannot consider surgical intricacies, surgeon experience or hospital-specific acute care standards [8, 20]. An essential first step in mitigating this inaccuracy, and a function currently missing from the ACS NSQIP surgical risk calculator, is the combination of $\mathrm{CPT}$ codes to build a case more representative of the actual procedure [32].

Our analysis of the risk calculator for calibration and discrimination followed a similar trend as LOS with all 12 outcomes properly calibrated in the NFF group and 8 of 12 calibrated in the FF group. As mentioned by Winoker et al., it is important to emphasize that few incidences of observed complications can contribute to low Brier scores [9]. This can perhaps be seen in outcomes such as renal failure, VTE, and UTI in our cohort. Interestingly, the risk calculator exhibited slightly better discrimination for outcomes in the FF group (4 of 12) than in the NFF group (3 of 12). One possible explanation for this phenomenon is the improved performance of models that exhibited higher levels of heterogeneity. As shown by Cohen et al., ACS NSQIP surgical risk calculator estimations, projected for heterogeneous populations of patients (those that undergo many different procedures and have a diversity of preoperative morbidities), score better for discrimination than risk projections for homogenous patient populations [9]. The risk calculator scored well for both calibrated and discrimination for 6 of 12 outcomes in the total study population. Our results demonstrating the risk calculator's accuracy for a portion of complications predicted are consistent with that of previous publications [21, 22, 33]; however, they are inconsistent with

Table 4 Brier score and ROC AUC for all complications calculated together

\begin{tabular}{|c|c|c|c|c|c|c|}
\hline \multirow[b]{2}{*}{ Outcome } & \multicolumn{2}{|c|}{ Without Free Flapa ${ }^{a, b}$} & \multicolumn{2}{|c|}{ With Free Flap ${ }^{a, b}$} & \multicolumn{2}{|c|}{ Combined $^{a, b}$} \\
\hline & Brier Score & ROC & Brier Score & $\mathrm{ROC}$ & Brier Score & $\mathrm{ROC}$ \\
\hline Overall & 0.0230 & 0.7790 & 0.0814 & 0.8520 & 0.0548 & 0.8590 \\
\hline
\end{tabular}

${ }^{\mathrm{a}}$ Brier scores of $<0.09$ and $\mathrm{ROC}>0.7$ (bold values) were considered accurate

${ }^{\mathrm{b}}$ Bold values meet or exceed their respective accuracy thresholds 


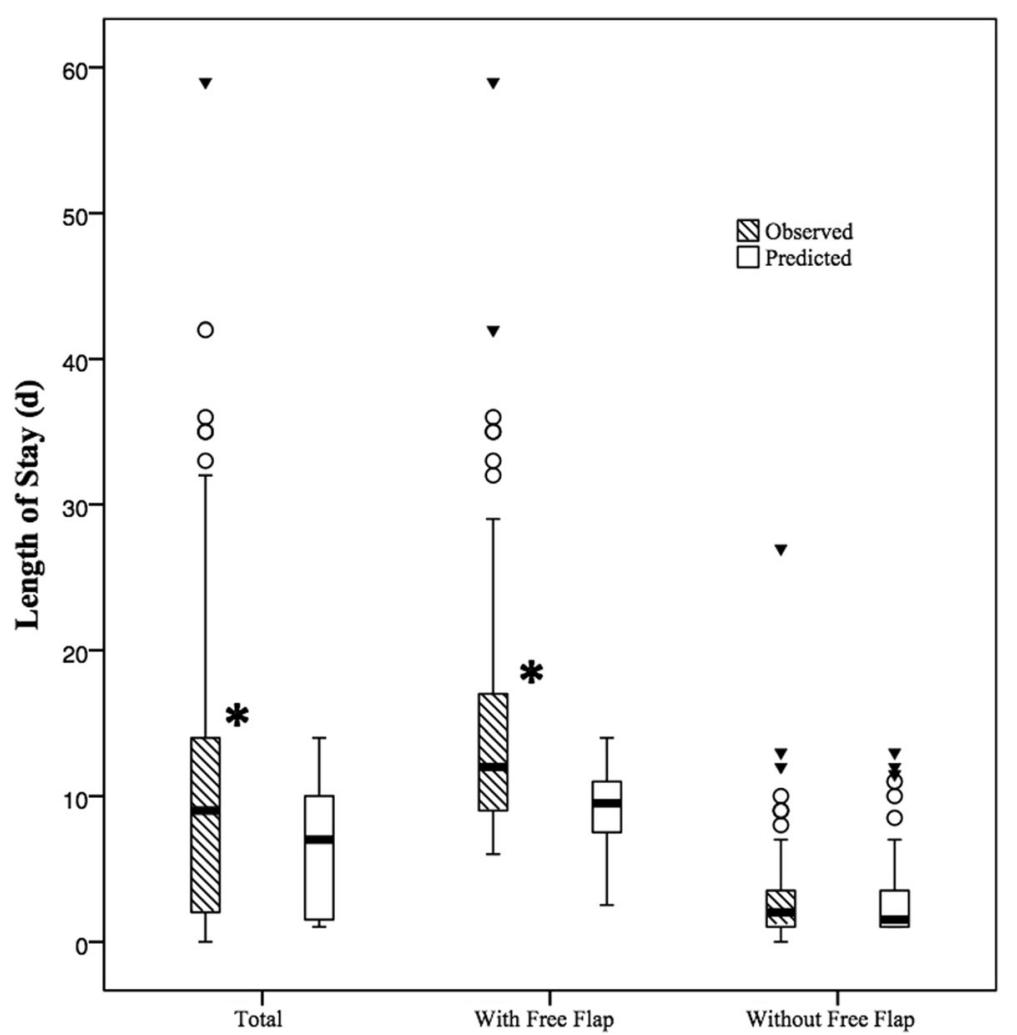

Fig. 3 Comparison between predicted and observed length of hospital stay for study population and free flap stratifications. Footnote: *Observed and predicted lengths of stay were found to significantly differ

other evaluations specific to the head and neck discipline. Two potential reasons for this discrepancy are differences in Brier score threshold values used and patient population heterogeneity. The former, explained in the discussion above, has resulted in previous studies considering acceptable scores as non-predictive $[16,17]$. The latter refers to previous studies evaluating only one head and neck surgical procedure and thus exhibiting lower levels of heterogeneity $[15,16]$.

Analysis by procedure type further substantiated the risk calculator's accuracy in our patient cohort, with 7 of 8 evaluated procedures showing good calibration and all calculable procedures meeting adequate discrimination levels. Potential reasons why the risk calculator could have shown poor calibration for laryngectomy, which scored just above the Brier threshold, includes relatively few patients $(n=5)$ having undergone this procedure and thus a potential for sampling error, and the relatively high incidences of T4 cancer $(100 \%)$ and prior radiation $(60 \%)$. Interestingly, of the procedures analyzed that met the Brier score threshold, composite resection with free flap and laryngopharyngectomy with free flap had the highest values. This relative decrease in calibration can potentially be attributed to the increased complexity associated with these surgeries. This is certainly supported considering the results of the free flap stratified analyses.

For the overall population analyses, in which all surgical procedures and complications were pooled together, discrimination and calibration computations met their respective thresholds this provides the strongest evidence in our analysis of the risk calculator's effectiveness in predicting complications secondary to head and neck surgery.

\section{Primary strengths}

We present the first comprehensive evaluation of the ACS NSQIP surgical risk calculator in the head and neck discipline in a Canadian setting. Furthermore, to the authors' knowledge we are the first publication on the risk calculator to stratify by microvascular reconstruction, a surgical procedure which is associated with longer OR times and increased complication rates. Finally, our findings demonstrate good performance of the ACS NSQIP surgical risk calculator in predicting postoperative complications in patients undergoing head and neck surgery without free flap reconstruction. Given these results, we regularly utilize the risk calculator to determine individual patient's risk of complications as a decision-support tool to evaluate the safety of the procedure and to provide more 
comprehensive preoperative counselling to patients and their families.

\section{Limitations}

We are primarily limited by the size of our study cohort $(n=107)$, having few patients with complications (27), and being a single institution retrospective review. The latter two limitations have been implicated by Cohen et al. as potential sources of inaccuracy when evaluating the risk calculator [11]. Specifically, they concluded that three criteria must be met to fairly evaluate the risk calculator: a patient cohort acquired from more than one care center, a minimum of 100 patients with an incidence, and sufficient cohort heterogeneity. Based on the diverse list of procedures reviewed, we believe the level of heterogeneity required has been met in our cohort.

\section{Next steps}

Specialty-specific surgical risk calculators have shown improved predictive accuracy over the ACS NSQIP risk calculator [12, 23, 32], suggesting development of a head and neck-specific risk calculator is warranted. One potential caveat of these additional metrics, as was pointed out by Cohen et al., is the potential for overfitting [11]. Application of surgery-specific risk calculators should be used only on the procedures and settings which they have been examined. To this end, a multicenter collaboration as part of the Canadian Association of Head and Neck Surgeons is planned.

The purpose of a risk calculator is to determine the potential complications our patients may endure based on patient comorbidities and the procedures planned. The ACS NSQIP surgical risk calculator predicts the major complications of any surgery; however, it does not predict complications specific to our patient population that impair quality of life and increase LOS including fistula development and free flap failure requiring a second free flap or locoregional flap. A specialty-specific risk calculator would address this shortcoming.

\section{Conclusion}

The ACS NSQIP surgical risk calculator has the potential to act as a quality improvement metric and aid in the informed consent process through preoperative planning and postoperative prevention of potential mortality and morbidity. Though data collected for the risk calculator was amassed entirely from American hospitals with the intended use of improving the standard of care within the American health care system, our results suggest the potential utility of the risk calculator for Canadian Head and Neck oncology. Specifically, judicious application of the risk calculator for head and neck surgical procedures that do not involve microvascular reconstruction is supported.

\section{Abbreviations}

ACS NSQIP: American College of Surgeons National Surgical Quality Improvement Program; AUC: Area under the curve; COPD: Chronic obstructive pulmonary disease; CPT: Current Procedural Terminology; FF: Free flap reconstructed patient group; LOS: Length of stay; NFF: Non-free flap reconstructed patient group; REB: Research ethics board; ROC: Receiver operating characteristics; ROR: Return to operating room; SSI: Surgical site infection; UTI: Urinary tract infection; VTE: Venous thromboembolism

\section{Acknowledgements}

The authors would like to acknowledge Michael Hong, BSc, for his contributions to the manuscript.

\section{Funding}

No forms of funding were acquired for this research.

\section{Availability of data and materials \\ Supporting data can be requested through contact with the corresponding author.}

\section{Authors' contributions}

All authors were involved with the conception and design of the study, analysis and interpretation of data, revising the manuscript, and have given final approval. Further to this PV was involved with the design, direction and writing of the manuscript, including figures and statistical analyses. MO was involved in developing and editing the manuscript, completing the statistical analysis and figures, and editing the manuscript. $\mathrm{KH}$ and DE were the attending surgeons of the patients analyzed in this study.

\section{Ethics approval and consent to participate}

Research ethics approval was obtained from the Sunnybrook Health Sciences Center Research Ethics Board (REB). The following REB number applies 2632017.

Consent for publication

Not Applicable.

\section{Competing interests}

The authors declare that they have no competing interests.

\section{Publisher's Note}

Springer Nature remains neutral with regard to jurisdictional claims in published maps and institutional affiliations.

Received: 17 August 2017 Accepted: 12 March 2018

Published online: 22 March 2018

\section{References}

1. Parikh P, Shiloach M, Cohen ME, Bilimoria KY, Ko CY, Hall BL, et al. Pancreatectomy risk calculator: an ACS-NSQIP resource. HPB. 2010;12:488-97.

2. Ramanan B, Gupta PK, Gupta H, Fang X, Forse RA. Development and validation of a bariatric surgery mortality risk calculator. J Am Coll Surg. 2012;214:892-900.

3. Gupta PK, Franck C, Miller WJ, Gupta H, Forse RA. Development and validation of a bariatric surgery morbidity risk calculator using the prospective, multicenter NSQIP dataset. J Am Coll Surg. 2011;212:301-9.

4. Nam RK, Kattan MW, Chin JL, Trachtenberg J, Singal R, Rendon R, et al. Prospective multi-institutional study evaluating the performance of prostate cancer risk calculators. J Clin Oncol Off J Am Soc Clin Oncol. 2011;29:2959-64.

5. About - ACS Risk Calculator. https://riskcalculator.facs.org/RiskCalculator/ about.html. Accessed 18 Jul 2017.

6. Velanovich $\mathrm{V}$. Testing the ability of the NSQIP risk calculator to predict laryngectomy complications. JAMA Otolaryngol Head Neck Surg. 2016;142: 979-80.

7. Winoker JS, Paulucci DJ, Anastos H, Waingankar N, Abaza R, Eun DD, et al. Predicting complications following robot-assisted partial nephrectomy with the ACS NSQIP universal surgical risk calculator. J Urol. 2017; https://doi.org/ 10.1016/j.juro.2017.04.021.

8. Vaziri S, Wilson J, Abbatematteo J, Kubilis P, Chakraborty S, Kshitij K, et al. Predictive performance of the American College of Surgeons universal risk calculator in neurosurgical patients. J Neurosurg. 2018; 128(3):942-7. 
9. Cohen ME, Liu Y, Ko CY, Hall BL. An examination of American College of Surgeons NSQIP surgical risk calculator accuracy. J Am Coll Surg. 2017;224: 787-795.e1.

10. McMillan MT, Allegrini V, Asbun HJ, Ball CG, Bassi C, Beane JD, et al. Incorporation of procedure-specific risk into the ACS-NSQIP surgical risk calculator improves the prediction of morbidity and mortality after Pancreatoduodenectomy. Ann Surg. 2017;265:978-86.

11. O'Neill AC, Bagher S, Barandun M, Hofer SOP, Zhong T. Can the American College of Surgeons NSQIP surgical risk calculator identify patients at risk of complications following microsurgical breast reconstruction? J Plast Reconstr Aesthetic Surg JPRAS. 2016;69:1356-62.

12. Slump J, Ferguson PC, Wunder JS, Griffin A, Hoekstra HJ, Bagher S, et al. Can the ACS-NSQIP surgical risk calculator predict post-operative complications in patients undergoing flap reconstruction following soft tissue sarcoma resection? J Surg Oncol. 2016;1 14(5):570.

13. Arce K, Moore EJ, Lohse CM, Reiland MD, Yetzer JG, Ettinger KS. The American college of surgeons national surgical quality improvement program surgical risk calculator does not accurately predict risk of 30-day complications among patients undergoing microvascular head and neck reconstruction. J Oral Maxillofac Surg Off J Am Assoc Oral Maxillofac Surg. 2016;74:1850-8.

14. Schneider AL, Deig CR, Prasad KG, Nelson BG, Mantravadi AV, Brigance JS, et al. Ability of the national surgical quality improvement program risk calculator to predict complications following total laryngectomy. JAMA Otolaryngol Head Neck Surg. 2016;142:972-9.

15. Prasad KG, Nelson BG, Deig CR, Schneider AL, Moore MG. ACS NSQIP risk calculator: an accurate predictor of complications in major head and neck surgery? Otolaryngol Head Neck Surg Off J Am Acad Otolaryngol-Head Neck Surg. 2016;155:740-2

16. Samson P, Robinson CG, Bradley J, Lee A, Broderick S, Kreisel D, et al. The National Surgical Quality Improvement Program risk calculator does not adequately stratify risk for patients with clinical stage I non-small cell lung cancer. J Thorac Cardiovasc Surg. 2016;151:697-705. e1

17. Teoh D, Halloway RN, Heim J, Vogel Rl, Rivard C. Evaluation of the American college of surgeons national surgical quality improvement program surgical risk calculator in gynecologic oncology patients undergoing minimally invasive surgery. J Minim Invasive Gynecol. 2017;24:48-54.

18. Massoumi RL, Trevino CM, Webb TP. Postoperative complications of laparoscopic cholecystectomy for acute cholecystitis: a comparison to the ACS-NSQIP risk calculator and the Tokyo guidelines. World I Surg. 2017:41:935-9.

19. Basta MN, Bauder AR, Kovach SJ, Fischer JP. Assessing the predictive accuracy of the American college of surgeons national surgical quality improvement project surgical risk calculator in open ventral hernia repair. Am J Surg. 2016;212:272-81.

20. Adegboyega TO, Borgert AJ, Lambert PJ, Jarman BT. Applying the national surgical quality improvement program risk calculator to patients undergoing colorectal surgery: theory vs reality. Am J Surg. 2017;213:30-5.

21. Hu W-H, Chen H-H, Lee K-C, Liu L, Eisenstein S, Parry L, et al. Assessment of the addition of hypoalbuminemia to ACS-NSQIP surgical risk calculator in colorectal Cancer. Medicine (Baltimore). 2016;95:e2999.

22. Peterson B, Ghahramani M, Harris S, Suchniak-Mussari K, Bedi G, Bulathsinghala $C$, et al. Usefulness of the myocardial infarction and cardiac arrest calculator as a discriminator of adverse cardiac events after elective hip and knee surgery. Am J Cardiol. 2016;117:1992-5.

23. le Nobel GJ, Higgins KM, Enepekides DJ. Predictors of complications of free flap reconstruction in head and neck surgery: analysis of 304 free flap reconstruction procedures. Laryngoscope. 2012;122:1014-9.

24. Klosterman $\mathrm{T}$, Siu E, Tatum S. Free flap reconstruction experience and outcomes at a low-volume institution over 20 years. Otolaryngol Head Neck Surg Off J Am Acad Otolaryngol-Head Neck Surg. 2015;152:832-7.

25. ACS Risk Calculator - Home Page. https://riskcalculator.facs.org/ RiskCalculator/. Accessed 25 Jul 2017.

26. Hajian-Tilaki K. Receiver operating characteristic (ROC) curve analysis for medical diagnostic test evaluation. Casp J Intern Med. 2013;4:627-35.

27. Hosmer DW, Lemeshow S. Applied logistic regression. 2nd ed. New York: Wiley; 2000.

28. Gerds TA, Cai T, Schumacher M. The performance of risk prediction models. Biom J Biom Z. 2008:50:457-79.
29. Steyerberg EW, Vickers AJ, Cook NR, Gerds T, Gonen M, Obuchowski N, et al. Assessing the performance of prediction models: a framework for traditional and novel measures. Epidemiol Camb Mass. 2010;21:128-38.

30. Bilimoria KY, Liu Y, Paruch JL, Zhou L, Kmiecik TE, Ko CY, et al. Development and evaluation of the universal ACS NSQIP surgical risk calculator: a decision aide and informed consent tool for patients and surgeons. J Am Coll Surg. 2013;217:833-842.e3.

31. Cohen ME, Bilimoria KY, Ko CY, Hall BL. Development of an American college of surgeons national surgery quality improvement program: morbidity and mortality risk calculator for colorectal surgery. J Am Coll Surg. 2009:208:1009-16.

32. Veeravagu A, Li A, Swinney C, Tian L, Moraff A, Azad TD, et al. Predicting complication risk in spine surgery: a prospective analysis of a novel risk assessment tool. J Neurosurg Spine. 2017;27:81-91.

33. Liu Y, Cohen ME, Hall BL, Ko CY, Bilimoria KY. Evaluation and enhancement of calibration in the American College of Surgeons NSQIP surgical risk calculator. J Am Coll Surg. 2016;223:231-9.

\section{Submit your next manuscript to BioMed Central and we will help you at every step:}

- We accept pre-submission inquiries

- Our selector tool helps you to find the most relevant journal

- We provide round the clock customer support

- Convenient online submission

- Thorough peer review

- Inclusion in PubMed and all major indexing services

- Maximum visibility for your research

Submit your manuscript at www.biomedcentral.com/submit
Biomed Central 